Manlio Cortelazzo,

Trieste - Padooa

\title{
TRACCE DELL'ANTICO DIALETTO VENETO DI PIRANO
}

Il Comitato dell'Atlante Linguistico Mediterraneo (ALM), dopo aver accettato d'includere nel piano dei suoi lavori un'inchiesta presso i pescatori di lingua slovena di S. Croce di Trieste (Sv. Križ pri Trstu) inchiesta egregiamente compiuta tra il 1966 ed il 1967 da T. Logar e S. Škerlj nel quadro dei rilievi per un grande atlante linguistico sloveno -, ha aderito anche alla proposta parallela di allargare le inchieste italiane, aggiungendo alla rete precedentemente stabilita un punto sulla costa istriana. La scelta è caduta su Pirano (Piran) per due motivi: la sua dislocazione geografica, come ultimo porto meridionale dell'area slovena, e la sua fisionomia dialettale, abbozzata dall'Ascoli (1873), illustrata fin dal 1900 dall'Ive, che includeva il piranese fra I dialetti ladino-peneti dell'Istria, esemplificata dal Parenzan attraverso 24 sonetti in vernacolo (il titolo della raccoltina - Del dialetto di Pirano. Saggio di Pietro Parenzan riveduto e annotato dal Conte Nicodemo Ton, Trieste, 1901 - non inganni) e indirettamente descritta dallo Scheuermeier, che visitò la cittadina nel maggio del 1922 per l'AIS (punto n. 368), e dal Pellis con una larga inchiesta diretta nel suo territorio (precisamente a Sicciole di Pirano), condotta nel 1926 e nel 1928 per l'Atlante Linguistico Italiano (ALI).

L'osservazione di un vecchio professionista residente a Pirano da molti anni, che l'antico dialetto (quello studiato dall'Ive, per intenderci), almeno nel centro, non è più parlato da decenni (lui stesso l'ha casualmente colto una volta sola nei discorsi di due vecchie popolane), è stata confermata tanto nel corso dei lavori di raccolta per l'ALM (29 marzo 4 aprile 1972), quanto con la richiesta diretta a più informatori, ai margini e al di fuori dell'inchiesta nautico-peschereccia, di notizie su questo tipo di dialetto arcaico, vagamente ricordato ed ancor più vagamente caratterizzato anche dai più anziani.

Occorre poi aggiungere che lo stesso dialetto prettamente veneto sovrapposto, a cui si era ridotto il piranese, ${ }^{1}$ è, a sua volta, in completo

${ }^{1}$ Cfr. B. Giraldi, Musa vernacola piranese (Versi inediti di Orazio de Colombani), «La Porta Orientale» V (1935), pp. 463-472: «In queste poesie vi è 
disfacimento, sia per la diaspora della compatta comunità, che l'usava, sia per i frequenti e vivaci contatti con l'esterno (le coste italiane per gli uomini di mare, Trieste, irradiatrice della koinè veneto-giuliana, per tutti), sia per l'influenza, all'interno, del contatto con i numerosi turisti e, più, per la larga ed incisiva penetrazione dell'italiano comune, diffuso specialmente attraverso le trasmissioni radio-televisive e l'insegnamento scolastico (esiste a Pirano, in lingua italiana, una scuola d'obbligo di otto anni col successivo ginnasio).

Ma, poiché il dialetto difficilmente conosce una morte repentina, completa ed assoluta, e anche dopo la sua scomparsa dall'uso vivo riesce ancora a lasciare lievi tracce in alcuni relitti, resistenti per capacità propria (fossilizzazione toponomastica, specializzazione semantica, appoggio fonetico) alle travolgenti ondate innovatrici, così è stato dato di cogliere anche nell'attuale parlata piranese, pur senza compiere un'apposita indagine, ma cercando di porre attenzione su alcuni particolari notati durante le conversazioni spontanee, pochi segni di una diversa e lontana situazione dialettale.

$$
* * *
$$

DITTONGAMENTI. - Al di fuori dell'inchiesta si è registrato piésa 'piccolo appezzamento di terreno' ( $p i e ́ \vartheta a$ in Ive 72) e da un informatore dell'ALM, poi titubante a confermare pubblicamente la variante, skuòta 'scotta' (nell'ALI 5192 skòta).

Sono i due soli esempi di dittongazione di è ed ò in posizione, che ricorda da una parte le condizioni del pavano, dall'altra soprattutto il rovignese (Ascoli 442).

Lo Scheuermeier aveva già raccolto per l'AIS:

II 235 'lievito': la fiésa (ma per un diverso informatore fésa con l'asserzione che »fiésa è slavo«; invece anche l'ALI 3998 'feccia (del vino)' e 5783 'feccia della birra' ha fièsa);

III 463 'vespa': la biéspa (come nell'ALI 4527);

VII 1399 'il mucchio di fieno': la tiésa (ALI 3638 'mucchi di erbe' o 3639 'di fieno': tiése, 3682 'bica': tiesa; tiéva in Ive $72<$ TENSA). Nel V sonetto del Parenzan tanto kuliéta) Ive 72, quanto quel ulì / Che 'l ha culièto 4 (<COLLECTUS: cfr. kuliédi, Barba Mìo per le pieze urlando 'l va (<PETIA) 12.

LE VOCALI ATONE FINALI. - Una sezione interessante del piranese riguarda alcuni fenomeni, che colpiscono le atone finali:

tutto il colore della nostra terra e del nostro vero dialetto veneto-istriano, non di quello vetusto di cui Piero Parenzan era il tipico rappresentante, ma di un dialetto - diremo - più attuale e consono ai mutamenti della vitas (p. 465).

Del resto il Colombani stesso $(1820-1873)$ dichiara i suoi versi dialettali ora in dialeto venezian o in persi veneziani, ora in dialetto veneto-istrian (ib., p. 470). 
a) $-i$ per -e di terza persona, ignoto a Rovigno e Dignano, ma non al polesano e al triestino (Áscoli 439, Ive 74);

b) $-i$ per -o di prima persona, con scarsi resti a Pirano e Rovigno, cospicui nel dignanese (Ascoli 437, Ive 75):

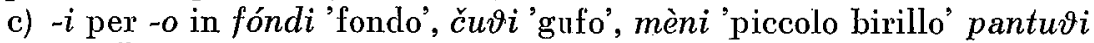
pantano' (Ive 75);

d) -e per -o in kólme 'colmo' (e 'comignolo'), hánte 'santo', réme 'remo', Karse 'Carso', piédene 'pédano' (lve 75).

Persistente è solo il caso a), appoggiato com'è alla salda tradizione triestina

bati él mar ALM 27

él mar sé rómpi ALM 28

pióvi ALM 90

lá spandi ALM 250

lé fbati '(le vele) fileggiano' ALM 360

él sérčo sénti él temporàl

e documentato, naturalmente, anche nei rilievi dell'AIS (p. es, IV 651: dòrmi) e dell'ALI (1287 'corre': el kóri; anche nòmi per nòme 1431 e tósi per tóse 6522 bis).

Dell'ultima serie s'è raccolto rème ALM 314 (come in ALI 5166) e Karse. ambedue registrati anche nel Rosamani; kólme 'tetto' hanno l'AIS V 864 e l'ALI 1519, come pure il Parenzan (Mi passo qua sul colme ore beate XVII 3).

METAFONESI. - Se non si è avuto modo di accertare la sopravvivenza di plurali metafonetici, già rari nell'AIS (VII 1309 bàmpeno 'il pampano', pl. bàmpini) e nell'ALI (3084 bókolo, bókuli 'boccio di rosa', 3335 nùvolo, nùouli), s'è avuto modo di constatare il persistere di un'oscillazione di $e$ e $o$ in atonia con l'avvicinamento più o meno chiaramente avvertito, a seconda degli informatori, ad $i$ e $u$, rispettivamente, e qualche ripristino ipercorrettivo:

skapolàr skapulàr 'evitare (le secche)' ALM 166,

ódega ódiga 'gangamo' ALM 476,

ma anche

buraska boraska ALM $103 \mathrm{e}$

murada morada ALM 228.

Cfr. arkombè arkumbè 'arcobaleno' AIS II 371 - arkumbé ALI 3337 - poléoola puliéoola 'gavocciolo' ALI 474, gotifìm 'bicchierino' ALI 797 ter guti/ìm ALI 797.

LE ASPIRATE. - Gli informatori più vecchi e più attenti alle condizioni dialettali mantengono ancora, a livello di sfumato ricordo, la memoria di un suono proprio dell'antico dialetto e poi definitivamente scomparso, un suono che essi definiscono 'gutturale' o 'aspirato', per cui, 
ad esempio, la prima persona del verbo avere si sarebbe pronunciata [hò], anziché [gò].

Era ancor vivo sulla bocca dell'informatore del Pellis (Odorico Petronio detto Freschi, settantunenne nel 1926), che osserva: «Dei suoni consonantici merita particolare attenzione il suono $h$ : è articolato leggermente e fugacemente; un' 'aspirazione'. Ed è proprio della parlata dei popolani fra popolani, come appunto del mio informatore, non di quella fra 'cittadini', che usava $\int$ e $s$... Il P. si compiaceva di questo uso della buona tradizione popolare, aggiungeva però rivolgendosi a me: - $i$ di anka.....

Dalle annotazioni del Pellis, dai suoi esempi, come:

$\begin{array}{llll}\text { 1089. } & \text { Che ora è? } & \text { ke órahe } h^{\prime} & V^{-}: \text {le } \\ \text { 1090. Sono le 22; } & \text { le le diéhe } & V^{\prime}: \text { diéfe } \\ \text { 1625. una settimana ùna hetimàna } & V^{\prime-} \text { : set- } \\ \text { 1626. un mese } & \text { un méhe } & V^{\prime}: \text { méfe } \\ \text { 1933. soldo } & \text { hòldo } & V^{\prime}: \text { sòldo, ecc.; }\end{array}$

da qualche verso del Parenzan

- Mariavè ! hele gnanca porcarie II 9

Dihème, hélo fora? - De la béla III 6

Hè de foje e fastughi un sgolatìo .. V V 6 (cit. anche de Rosamani 466)

Dài, la hè qua I Sbrighève benedete ! - XVI 1

(nell'AIS il fenomeno non si rileva), appare chiaro che l'applicazione esemplificativa suaccennata è deviante e che il fenomeno interessa piuttosto il passaggio $s, f>h$ (alternante con $\vartheta$ ), segnalato dall'Ive 78 ( $\mathrm{hal}$, hol, hóldo e un $\vartheta o ́ l d o . .$.$) e riscontrato anche del Rosamani 467$ «nella pronunzia dei vecchi piranesi», con la citazione di hope = sope 'zuppe' (figado co le hope di biga; cfr. Ive l.c. hópa 'pane inzuppato').

La sostituzione con la sibilante è, oggi, comunque, totale e assoluta, almeno nella cittadina.

LE AFFRICATE. - Ancora avvertibile e resistente, invece, contro lo stesso modello veneziano, che esclude oramai l'affricata sorda [z] dal suo sistema fonetico, è la conservazione della indebolita componente dentale. Non più, dunque, $[z]$, ma non ancora $[s]$.

Qui trascriviamo ${ }^{t}$, anche se nell'ALM apparirà la sola $s$ per l'irrilevanza fonematica e lessicale della lievissima occlusiva precedente, appena percettibile:

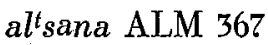

bonatsa ALM 9, 77, 78, 110 (ALI 5098: bonása)

bratsèra ALM 210

matsiòla ALM 262

pasaîtso 'tondino di ferro' 
radatsa ALM 302

tertsarióli ALM 363,

mentre il Rosamani propende ora per $s$, ora per $z$, «secondo che l'abbiamo sentita» (XIVVI: ma l'esposizione è un po' confusa). Nell'AIS:

II 249 kasóla 'cazzuola'

III 551 la masòka 'la mazza di legno',

che è esito finale, mentre alla fine del secolo scorso la soluzione più frequente dei nessi $\mathrm{TJ}, \mathrm{CJ}$ era ancora $\vartheta$ e più di rado $z$ (Ive 77).

PALATALIZZAZIONE. - Il Rosamani testimonia gnegarse solo nel dialetto di Pirano, che annovera altri esempi (tutti d'effetto analogico, come ritiene l'Ive 76 ?) di $n>\tilde{n}:$ Deboto, Fina mia, la s'ha gnegado, Parenzan XI 14. Anche l'ALI, accanto a ñéspola (1997) e ñéspolàro (3891), ha ñéga $V^{-}$: néga (3016 quater) e ñegado con la variante niegado (5224) che possono rendere ragione della palatalizzazione.

Ancora vitale: n'egado 'annegato' ALM 206.

ST J DA SK J. - Anche il fenomeno più tipico del vecchio dialetto piranese, antico e isolato, perché collega Pirano solo con Grado, dove egualmente appare di tenace vitalità, cioè l'esito sti $<\mathrm{SK} J$, finora ritenuta caratteristica toscana (Rohlfs I $\$ 190$ ) e bolognese (Coco), come il parallelo, ma meno frequente, $\int d i<\mathrm{SG} \mathrm{J}$, è stato colto nell'inchiesta dell'ALM, che, se da un lato registra la forma recenziore skiuma (n. 19), dall'altro trasmette ancora pustía dé trainàr 'la polpara' ALM 466 (= ALI 5299 bis e Rosamani 842) e stiame 'squame' ALM 515.

Non sarà superfluo citare almeno gli esempi d'iniziale offerti dal Rosamani:

Pirano Grado altri luoghi

fdienza

$\left\{\begin{array}{l}\text { /dionfada } \\ \text { fdionfado } \\ \text { fdionfo (ALI } 455 \text { e 5246) }\end{array}\right.$

fstiafa (AIS IV 728)

\{stiafo (ALI 382)

stiama (ALI 5335)

stiavina

fdionf $\hat{a}$

stiafogiao

stiaoo

stiera

stieto

stiazada (Momiano)

Sstiera (Parenzo, Orsera)

\{stierar (Capodistria)

stieto (Fiume) 


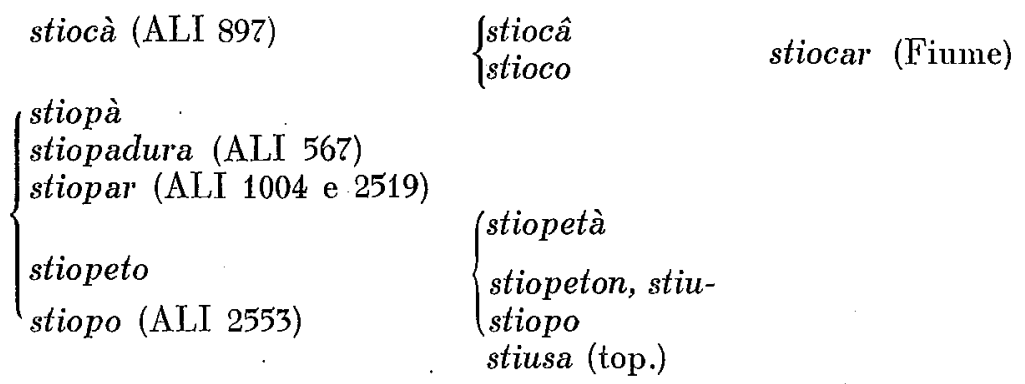

Non rilevato dall'Ascoli, non dall'Ive, ha attirato l'attenzione di Vidossi recensore dell'operetta di Parenzan («Archeogr. Triest.» N.S. XXIV, 1902, pp. 192-193), dove il tratto si manifesta apertamente

- Su per zinquina 'lora ! - fa una stiama I 4

- Presto la stiopa, che diseu mo, mama ? I 8 Po' fazo la polenta e una sdionfada IX 3.

E si aggiunga, dall'ALI:

4168 'vischio' : višco $l^{r^{-}}$: vistio

4592 'panie' : vistiade

5460 'ganghero' : mastio

(ma 1071 'coperta' : sčavina)

e l'AIS III 620 ha sì musčo 'muschio', ma da un secondo informatore mustio.

GLI INFINITI. - Comparando i dialetti istriani di Pirano, Rovigno e Dignano, l'Ascoli 436 notava la loro concordanza nella caduta di $-r$ dell'infinito col conseguente ridursi a $-i$ dell' $-e$ atona risultata finale nei verbi in -ERE. Aggiungeva: «Nei dialetti di Pirano. e Rovigno, le forme troncate oggi però si mescolerebbero con le veneziane, provedute del -7 »).

Ad un secolo di distanza la situazione non è ancora risolta, nel senso che, se pure, sospinte dagli esempi, oltre che veneziani, ma più triestini ( Riguardo all'infin. -are $>-a$ ho notato che i giovani tendono a ritornare a -àr ( kantàr), per influsso del triestino e della scuola» Pellis), anche italiani, le forme con $-r$, quando non addirittura con $r$ re, siano forse prevalenti nell'ordine della frequenza d'uso, purtuttavia gl'infiniti ridotti sono ancora sentiti come peculiari del piranese e strettamente caratterizzanti il dialetto:

nudà, nudàr (ALM 409 e 410), nudàre (cfr. «coesistendo talvolta le

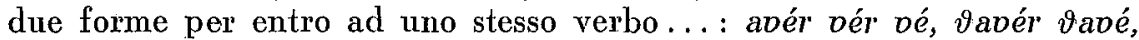
dolér dolé dulé» Ive :7)

la luna lé in kalà ALM 126

véndi 'vendere' ALM 431 
Nell'ALI:

$$
\begin{aligned}
& 913 \text { 'bollire' } \\
& 1304 \text { 'leggere' } \\
& 1769 \text { 'stirare' } \\
& 3225 \text { 'raccogliere' }
\end{aligned}
$$

nell'AIS:
: buì

: $l e^{h} \boldsymbol{i}$

: sopresà

: kuliéfi (< COLLIGERE);

: nàsi

: morì

: spudà

: tòsi,

oltre ai sostantivi, che negli altri dialetti veneti hanno pure -re:

$$
\begin{array}{ll}
\text { II } 331 \text { 'mercole(di)' } & \text { : mèrko } \\
\text { II } 333 \text { 'vener(dì)' } & \text { : vène, }
\end{array}
$$

entrambi citati anche dall'Ive 74 e 77 e raccolti altresì dall'ALI 63 e 65.

IL PRESENTE. - Un'oscillazione non equilibrata fra le due desinenze della terza persona del presente -éa (vitalissima all'epoca dell'Ive $83) \sim-a$ è ancora in qualche modo testimoniata nel dialetto, che mantiene tuttora almeno torgoléa "intorciglia'.

Dall'AIS: VI 1061 mulinéa 'rumina', VII 1117 fñagoléa 'miagola', VI 1124 kokoléa 'chioccia'; dall'ALI: 483 tartaèa 'balbetta', 3380 piuvifinèa 'pioviggina' (anche nell'Ive 83: piopidinéa, mentre l'analoga domanda 92 dell'ALM ha avuto, con l'infinito, una risposta elusiva:piovifinà), 5283-5284 inkatramèa 'spalmano catrame'.2

IL LESSICO. - Un gruppetto di parole straniere, tutti tedeschismi (notevole il loro addensamento intorno alla terminologia del carro), ${ }^{3}$ tranne alcuni slavismi, diffusi nell'intera area istro-giuliana (Rosamani) e slovena, ${ }^{4}$ è racimolabile nell'ALI:

ALI 832. pluča 'bottiglia scaldaletto': ted. dial. Plutzer;

1610. flai 'freno' e 3614 'martinicca': ted. Schleife;

4495. Jmìr 'grasso per carri': ted Schmiere;

4505. firùk! 'indietrol': ted. zurück;

5497. ago àkerle 'uncinetto': ted. Haken (cfr. ID XVII, 1941 pp. 131 e 232);

5599. pèstrina 'bambinaia': slov. pestrna;

5758. pek 'fornaio': ted. dial. Beck;

5880. klanfa 'grappa (di ferro)': ted. dial. Klampfe;

6042. kruka. 'picchiotto': ted. ant. krucka (ted. Krücke);

2 Pex il fenomeno si veda la grammatica storica del Rohlfs II $\$ 526$ - queste forme, già notate nel veneziano antico, si conservano fino ad oggi nel veneto periferico, anche se in lotta con le concorrenti meno lontane dal tipo italiano (cfr. J. Mazzarolo, Contributo alla sintassi del dialetto asolano, Padova, 1969 -1970, pp. 169-172: tesi di laurea inedita).

${ }^{3}$ W. H. Striedter-Temps, Deutsche Lehnmörter im Slovenischen, Wiesbarlen, 1963.

${ }^{4}$ Cfr. G. Pinguentini, L'elemento straniero nel dialetto triestino, in appendice al Nuopo dizionario del dialetto triestino, Bologna, 1969 pp. 372-373. 
6476. klaftar 'tesa (parı a 6 piedi)': ted. Klafter;

6494. funto 'libbra': ted. Pfund;

l'inchiesta per l'ALM e le conversazioni collaterali hanno permesso di raccogliere, oltre a voci inglesi d'uso internazionale nella marina, come blak 'catrame': ingl. black, lòk 'solcometro': ingl. log, dòk 'darsena (per pulire lo scafo)': ingl. dock, pispai, ed anche fìspai 'legno scuro da costruzione': ingl. pitch-pine 'pino americano o resinoso', qualche altro residuo mitteleuropeo:

petès 'alcool', dim. petesina; petesòni 'grandi bevitori' (abbastanza diffuso: Rosamani 772): fr. ? o slavo («Arch. slav. Phil.» XXVI, 1904, p. 413)? o it. gerg. («Archeogr. Triest.» XXX, 1905, p. 161)?;

fèmisa 'bina di pane', ora in disuso tanto nell'Istria, come in Friuli (Rosamani 999): ted. Semmel, passato attraverso lo sloveno, che ha munito žemlja del suff. diminutivo -ica (žemljica);

fima 'freddo intenso', ma in uso scherzoso; anche a Trieste (Rosamani 1257): slov. zima;

flàif 'freno dei carri', già visto;

spaker 'cucina economica', di tutta l'Istria (Rosamani 1060): ted. Sparherd;

strusa 'filone di pane' (Rosamani 1112); ted. dial. Strutz (in slov. štruca). Qualche altro elemento lessicale più o meno notevole:

bàlega s. f. 'il giro della rete da posta' ALM 483. Il Rosamani 59 registra bàlego con diversi significati, tra cui, per Pirano, 'carniere', 'borsa, fagotto' (da Ive 85) e 'reticella a forma di sacco'.

baléna s.f. 'medusa' ALM 773: per quanto possa sembrare strano il significato è stato confermato da più fonti; del resto anche Rosamani 59 lo registra (ma solo a Pirano, probabilmente dall'ALI 5391).

baón (a-) loc. avv. 'voga di bratto' ALM 320. Cfr. baóna 'traccia, lasciata dal remeggio a poppa' Ive 85 (col riallacciamento a bava, cui maggiormente aderisce l' a bavón 'remare di poppa' dell'ALI 5279). frabo s. m. 'piccolo pesce, nero come il carbone': lett. 'fabbro'.

grankàdole s.f.pl. 'specie di conchiglie, che hanno un granchio nell'interno', cioè il 'bernardo l'eremita' (EUPAGURUS BERNHARDUS L.).

gròpo dé san nikolò s. m. 'nodo piano' ALM 373. - Denominazione isolata e légata ad una leggenda agiografica: mentre $\mathrm{S}$. Nicolò navigava gli si spezzò l'albero dell'imbarcazione; allora congiunse i due pezzi con una fune, 'inventando' il nodo piano.

karme s.f.pl. 'gorgonie' ALM 777, confermato dall'ALI 5331 ('quercia marina': karme).

kaùla s. f. 'grancella pieghettata' ALM 756, equivalente alla kaùrla 'piccolo granchio' dell'Tve 86 . Se voce neogreca, per il piranese vale, 


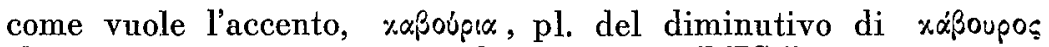
che si conserva, invece, in sardo e pugliese (DES I 325).

kondóta s. f. 'viaggio (fuori casa)': kuaranta ğórni dé kondóta a Póla. krità v. 'scricchiolare': lé karme lé krita ALM 777 (cfr. Parenzan XII 10: Sui telèri el brunzigo va critando). Dall'ALI 992 abbiamo '(il pane) crocchia': al krita.

kurìa s.f 'cèca' ALM 641, ma l'immagine deriva dal significato propirio 'stringa delle scarpe', che anche il Rosamani 282 registra per $\mathrm{Pi}$ rano, dall'ALI 648: kurìe 'laccetti (di cuoio)', poi sostituite dalle spigéte (di filo).

òrkole s. f.pl. 'torricelle comuni' ALM 720, proprio, secondo il Rosamani 706, di Pirano e Capodistria. - L'inchiesta dell'ALI 136 dà ad órkola il significato di 'ricciolo (dei capelli)'.

péa s.f. 'osso di seppia' ALM 704, come nel veneto, secondo l'Ive 88, che dà alla voce piranese il senso di 'corazza del granchio' (anche in Rosamani 752). Da PILLEUM '(copricapo di) feltro', che ha avuto un'ampia diffusione con significati adeguati al primitivo nucleo semantico ('copertura, buccia, pellicola, muschio, mallo della noce, riccio della castagna' e così via: REW 6504. e DEI IV 2822). Anche a Trieste pea 'Maia verrucosa', a Muggia pèie 'granziebole pice col baro sora' (Rosamani), e a Grado péğa 'grancevola femmina', mentre il maschio è il peğón (ALM). Per l'evoluzione fonetica ci si richiami a Vidossi Dial. triest. n. 111 (l i ama dileguare dopo e) e si confronti mèo 'meglio' (ALI 2335; $E$ in questa mèo. Parenzan III 3), pèo '(ci)piglio' (S'anca la me fa el pèo, id. VII 7), famèa 'famiglia' ALI 1465, famèo 'famiglio' ALI 4393, méo 'miglio' (cereale) ALI 3672.

pianèri s. m. pl. 'cercini per posare i pesi sul capo'. Propriamente: 'paniere' (cfr. Rosamani 776).

refósko s. m. 'ottima qualità di vino rosso' (cfr. per l'ampia diffusione Rosamani 868).

séri s. m. pl. 'tentacoli' ALM 703 e 712, incontrato anche dal Rosamani 1007 solo nel piranese, mentre le altre inchieste dell'ALM ne individuano due serie compatte, una veneto settentrionale (Grado e Muggia), l'altra che va da S. Benedetto del Tronto (čirri) fino a Taranto (čirrë) attraverso Pescara, Ortona, Vasto e Molfetta.

firnado p. p. 'pranzato': modo tipico dei vecchi (ankùo vu vè firnado): cfr. dòpo firnà 'pomeriggio' AIS II 339 e firnà 'pranzare' V 1029, isolato, con lo farnà (p. p. farnào) di Grado, in tutta l'area dialettale veneta. Nell'ALI, solo difnà 'pasto del mezzodì' 106, di/né? 'pranzate?' 938.

skòdeno s. m. 'tipo di abete: con la sua scorza si tingevano le reti', come ad Albona (Rosamani 976). È lo scotano.

flàveno s. m. 'alloro', come nell'ALI 4076 e 5755: una delle molte varianti istriane (Rosamani 531). 
Imartèla s. f. 'nome di pianta': il 'bosso' (?).

spontà s.f. 'puntura' (di pesce spinato) o anche 'iniezione': Rosamani 1077 sponta.

taranài s. m. pl. 'chele' ALM 766. Forma isolata in confronto al tananài, che Rosamani 1136 registra per Pirano e Rovigno (qui raccolto anche da M. Deanović per l'ALM).

tasádo p. p. 'morso' ALM 765, da tasà 'tagliare a pezzi' (sé tasava ALM 035), tasâ 'tritare' a Dignano, tazar 'tagliuzzare, tritare' a Trieste (Rosamani 1143, che dà tazâ 'tormentare, aguzzinare' anche per Pirano); nell'ALI 5659 el tasa 'trita' e 5665 tasaròla 'tagliere'.

tórgolo agg. 'sporco torbido'. - Dall'AIS V 1039 risulta che il piranese tòrgolo 'torbido' ha precisi riscontri col gradese e con altre varianti friulane (cfr. anche Rosamani 1165); aggiungi '(acqua) torbida': tórgola (ALI 3174).

\section{Ponzetek}

\section{SLEDI STAREGA BENEŠKEGA DIALEKTA V PIRANU}

Avtor meni, da je sedanji v Piranu govorjeni beneški govor dokaj drugačen od tistega starega, pravega benešlkega, ki ga je bilo moč slišati pred desetletji. Kolikor tega prvotnega govora ni uničila izselitev, ga je načela in ga uničuje moderna doba: stalen kontakt s pogovorno italijanščino, množična občila in seveda šola. Nekaj ostankov starega dialekta pa je moč ugotoviti v fonetičnih ostalinah (diftongi, nenaglašeni vokali $v$ absolutnem izglasju, metafonija, aspirirani glasovi), $\mathrm{v}$ glagolskih oblikah, in seveda $\mathrm{v}$ besednjaku, ki pozna mnogo slovenskih in nemških besedí (pestrina, zlaif, zmir, pek, klanfa. zemisa), beneščini v Benetkah seveda neznanih. 